\title{
Soft Pneumatic Actuator with Adjustable Stiffness Layers for Multi-DoF Actuation
}

\author{
Amir Firouzeh, Marco Salerno, and Jamie Paik ${ }^{1}$
}

\begin{abstract}
The soft pneumatic actuators (SPAs) are a solution toward the highly customizable and light actuators with the versatility of actuation modes, and an inherent compliance. Such flexibility allows SPAs to be considered as alternative actuators for wearable rehabilitative devices and search and rescue robots. In applications that require a high compliance for safety and a fluid interactivity, the SPAs material-based softness returns an inherent back-drivability. One of the main challenges that limits the wide application of SPAs is in the complexity of miniaturizing the actuators and embedding additional degree of freedoms (DoFs) in a single actuator. We present a novel design and fabrication method of a SPA with different modes of actuation using adjustable stiffness layers (ASLs). Unlike conventional SPA designs where one independent chamber is needed for each mode of actuation, here we have a single chamber that drives three different modes of actuation by activating different combinations of ASLs. By using customized micro heaters and thermistors for controlling the temperature and stiffness of ASLs in increments, we considerably broaden the work space of the SPA actuator. Here, a thorough characterization of the materials and the modeling of the actuator are presented. In the conclusion, we propose a design methodology for developing application specific actuators with multi-DoFs that are light and compact.
\end{abstract}

\section{INTRODUCTION}

Soft actuators are becoming one of the most appealing actuation possibilities in robots that have to deal with complex interaction conditions. Inherent compliance of the materials, adaptability of soft systems to unknown constraining conditions and back drivability are some of the advantages that make soft pneumatic actuators (SPAs) good candidates for many challenging tasks [1]. The versatility of the actuation modes, the ease of design and fabrication of application specific actuators, and the low cost of SPAs accelerate the iterations necessary in developing robotic systems. Recent advancements in rehabilitative devices [2], [3] and in mobile exploratory robots [4], [5] that use SPAs have clearly shown the potentials of SPAs in robotic application. Moreover, the latest achievement of mobile robots with embedded compressor unit and on-board electronics [4] confirmed the possibility of using these actuators even in untethered applications where the overall size and weight is confined.

In the conventional design of the actuators, the actuation of a single chamber can provide a system with a single motion based on the chamber design and the material used. One of the most typical drawbacks regarding the application of these pneumatic actuators in complex systems (requiring many

This work was supported by Swiss National Center for Competence in Research in robotics.

${ }^{1}$ The authors are with Reconfigurable Robotics Lab at EPFL, Lausanne. Jamie.paik@epfl.ch
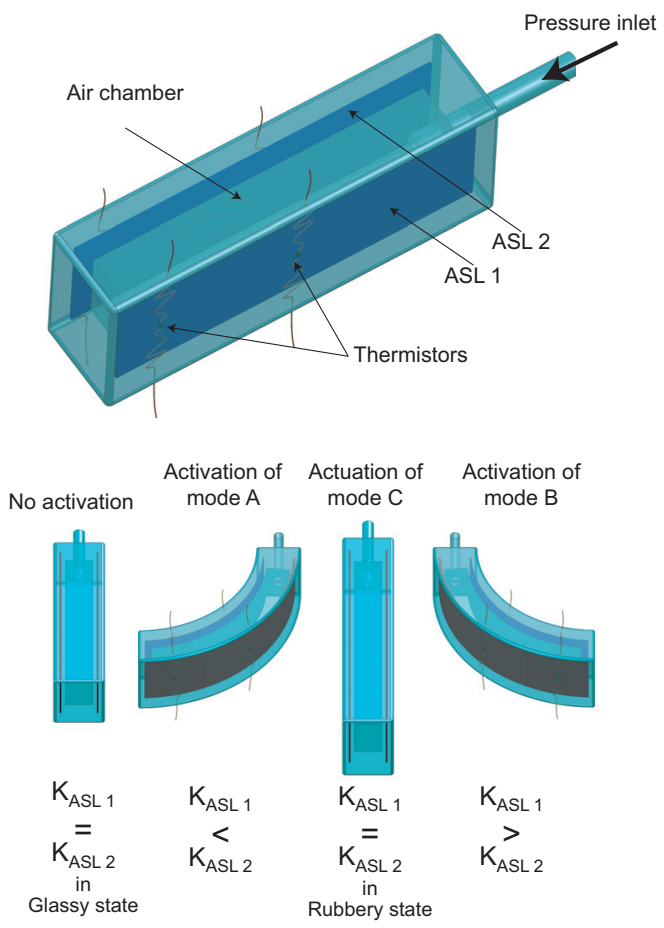

Fig. 1: A SPA with one chamber and two ASLs. The actuator can be actuated in 3 different modes: bending toward the opposite directions (mode A and $\mathrm{B}$ ), and elongation (mode C). The activation of different modes is carried out by controlling the stiffness of the two ASLs $\left(K_{A S L}\right)$.

degrees of freedom) is the encumbrance and complexity resulting from the use of numerous tubes, valves, regulators and other components needed to control the pressure within the chambers. The miniaturization becomes a challenge in such a system, given the complexity of the actuation for each chamber with independent pressure input. One method to tackle this problem is activating different modes of actuation on demand. Mechanically adding external layers for confining the deformation in specific segments as suggested in [6], [7] is an intuitive method to solve this problem. This is effective but requires modifying the hardware for changing the mode of operation. An alternative is to adapt variable stiffness components in the actuator that allows us to do the same without changing the hardware.

Vacuum induced granular jamming is one of the most employed principles for directing the central actuation to a desired point of application by changing the stiffness in different segments. In this method, chambers filled with particles can be stiffened through application of vacuum. This concept has been successfully applied in different 


\section{CONFIDENTIAL. Limited circulation. For review only.}

robotic applications [8]-[10]. The main remaining hurdle in using this method is the need for tubing and activation valves for each individual jamming chamber. An alternate method, which is less demanding in auxiliary equipment, is using elasticity change of thermoplastic polymers around their glass transition temperature. The Shape Memory Polymers (SMP) are one of the most preferred choices in such applications since they have the advantage of having more recovered deformation in the hot state and hence inducing a smaller residual strain. Researchers exploited the elasticity change in SMP coupled with a McKibben actuator for fixing the configuration of the actuator [11] and, in a bending version, for changing the range of motion and point of actuation [12], [13]. In this research, we aim to use the combination of SMP and customized pneumatic actuators in achieving different modes of operation in a SPA with a single chamber. Such a design reduces the complexity of pneumatic systems, allowing their miniaturization and improving their portability. Fig. 1 presents the proposed concept in which the actuation mode can be decided by controlling the stiffness of the chamber walls. By adjusting the stiffness of the walls, this actuator with a single chamber can deform in three major modes (bending in opposite sides and elongation). The adjustable stiffness layers (ASLs) in this design are composed of SMP embedded in silicone rubber.

The main contributions of this work are:

- Embedding the SMP layer in the silicone which solves the problem with the unrecoverable strain [14] in SMP thanks to the elasticity of the silicone.

- Expanding the work space of the actuator (beyond the major modes) by changing the stiffness of ASLs in increments thanks to the embedded thermistors which were used for closing the temperature loop.

- Material characterization and design process which paves the way for designing application specific SPA with ASLs.

In the next section, we will present the overall design of the ASL, its fabrication process, and report on its stiffness change with temperature. Next in Section III, using the characterization results for ASL, we will design a SPAs with ASL to reach a desirable range of motion and estimate its performance. We will also present briefly the fabrication process of the SPA with ASL in Section III. In Section IV, the results of the characterization tests on SPA with ASL will be presented and compared to the expected performance. Finally, in Section V, the conclusions of the work and the future steps will be presented.

\section{ASL DESIGN AND CHARACTERIZATION}

To introduce a guideline for designing an application specific SPA actuator with embedded ASLs, we need to characterize the stiffness of the components and their dependency on temperature. Mechanical properties of SMP including its recoverable range of deformation, the stressstrain characteristics and its dependence on the temperature are well studied [15]. The test conditions in these studies are usually very well controlled and an external heating elements ensures a uniform temperature distribution on the sample. In an actual application however, there will be a considerable temperature gradient over the entire area of the SMP. So, the characterization tests are necessary for understanding the behavior and determining the design parameters. In this section, first, the test sample design and fabrication process are presented. Then, the test setup used in characterization is introduced and finally, the results of the characterization tests will be presented.

\section{A. Test sample design and fabrication}

To design the SPA for reaching a desired range of deformation or force, we need to characterize the elasticity of different layers and materials in SPA. Since the recoverable strain of the SMP sample is higher when it is embedded in silicone rubber, it is necessary to characterize the ASL layer as a whole (since the performance of SMP on its own would be different from its performance when embedded in silicone). Further, to have a measure of the force in the two walls of the chamber without ASL, we will characterize a silicone rubber sample. Here, we present the design and fabrication of the ASL sample. The silicone sample is essentially the same just without the SMP layer.

Using external heating (as done in [16] and [11]) does not allow independent activation of the ASLs and embedding the heaters would be necessary to activate different modes in a SPA. Embedding conductive wires [13] in SMP layers was shown to be an effective method for integrating the heating element even if it can be considered feasible just for simple designs where routing the wire is not too difficult. A more attractive solution allowing the possiblity of miniaturization and segmented heating is to use stretchable heating elements. Here we use a stretchable heater made of patterned metalpolyimide laminate that can be processed in large areas with very high accuracy [14]. In the heater presented in Fig. 2, a mesh pattern that is cut through all layers provides the stretchability. The mesh pattern is designed on top of the main heater pattern which is in the shape of a serpentine path (to increase the resistance). As presented in Fig. 2, the heating area of the heater is longer than the stretchable area. The reason for the two unstretchable side sections of the heater is to have a more uniform temperature distribution along the stretchable section of the SMP layer. Next, the outline of the sample is cut according to the desired pattern. A detailed report on the fabrication process of the SMP layer with embedded heater can be found in [14]. We have embedded three thermistors (SMD 0402 from Vishay) in the test sample one in the middle of the heating element and two on the border of the stretchable area to monitor the temperature during tests and tune the driving voltage accordingly. After attaching the thermistors through heating, the SMP layer is placed in a mold and is embedded in the silicone rubber.

There are two openings on each side of the SMP layer in Fig. 2 (other than the holes for clamping the sample to the setup). These openings are designed to make silicone connections inside the SMP layer in the integration process. 


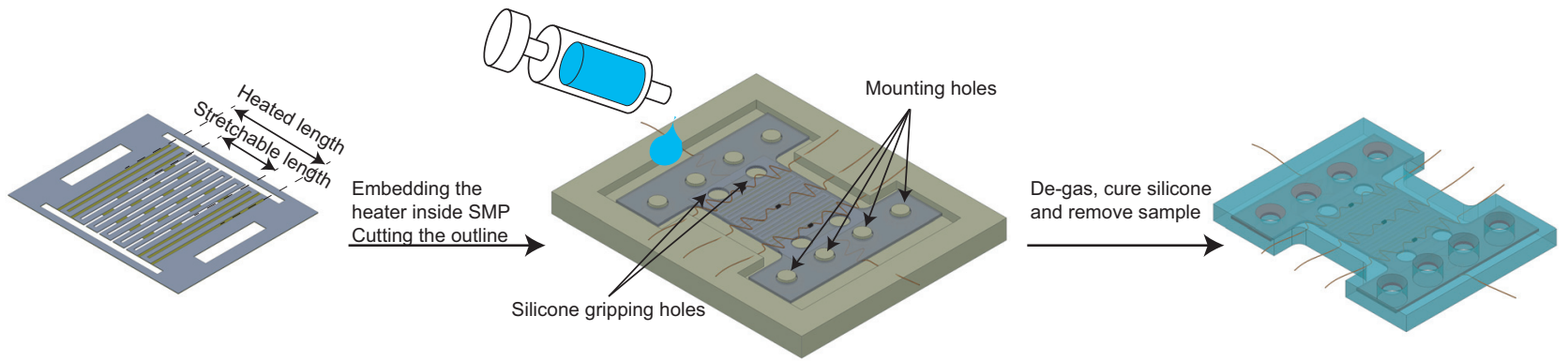

Fig. 2: Fabrication process of the ASL sample. The process starts with the fabrication of the heater. It composed of a serpentine path that makes the heater and an stretchable mesh pattern in the middle. Next, the heater is embedded in SMP using a heat press and its outline is cut using a laser micro machining tool (details on the fabrication of this part can be found in [14]). Three thermistors are attached to the SMP layer for monitoring its temperature. Next, the SMP layer is placed in a mold and is embedded in silicone rubber to produce the final sample. For clarity, the dimensions in the schematics are not to scale.

Since the adhesion between SMP and silicone is very weak, this method has been used as a mean for transmitting the force necessary for deforming the SMP in the pneumatic actuator. The cross section of the columns should be large enough so the material can bear the shear caused by the force according to the following equation:

$$
\frac{F}{2 \frac{\pi d^{2}}{4}}<0577 S_{p} / n_{s}
$$

In this equation, $F$ is the force needed for deforming the SMP sample, $d$ is the diameter of the silicone column in SMP, $S_{p}$ is the ultimate strength of the silicone rubber and $n_{s}$ is the safety factor. Also the column should resist against bearing:

$$
\frac{F}{2 t_{S M P} d}<S_{p} / n_{s}
$$

In this equation, $t_{S M P}$ is the thickness of the SMP layer. Based on the initial test on a SMP sample, the force we need to elongate the active sample (at high temperature) to twice

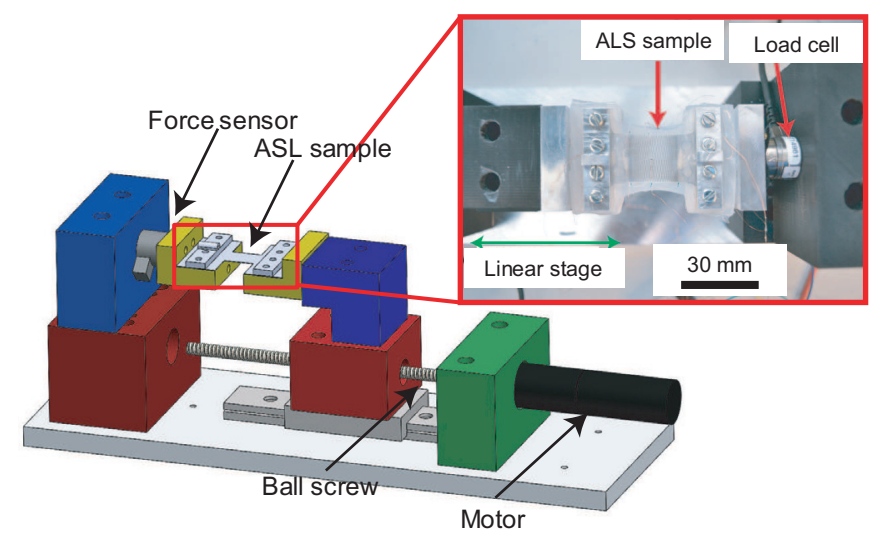

Fig. 3: Stress-strain test setup. The temperature of an ASL strip (as the test sample) is controlled through feedback from the thermistors either using an embedded heater or external heaters. The linear stage applies a series of programmed deformations at different temperatures while the load cell records the applied forces.

its initial length is around $8 \mathrm{~N}$. A strain of $100 \%$ is more than the expected work range in our case and a design based on it provides a further safety margin. Based on (1) and (2), the openings in the SMP layer were designed to be $4 \mathrm{~mm}$ in diameter which gives us a safety factor of 1.6.

\section{B. Test setup}

To characterize the stress-strain relation of the ALS in different temperatures, we have designed a setup that automatically performs a series of characterization tests in different temperatures and elongation ranges. Fig. 3 presents the test setup and a sample in test. The temperature is monitored and a proper input voltage is applied, to either an embedded heater in the material or external haters in the chamber which is surrounding the test sample, for keeping the desired temperature during the tests. The deformation is applied using a spindle drive from Maxon motors with $10 \mu \mathrm{m}$ accuracy. The force is measured with a Nano 17 force sensor with $3 m N$ accuracy.

\section{ASL performance characterization}

Fig. 4 presents the characterization test results for a silicone sample, and for an ASL sample in active (in high temperature) and inactive (in low temperature) states. The temperature used in active tests was set at $110{ }^{\circ} \mathrm{C}$ ). This temperature set point is necessary to ensure that the whole SMP layer surpasses the glass transition temperature of $55^{\circ} \mathrm{C}$ ). In the characterization of the ASL, we first cycled the inactive sample 50 times, then cycled the activated sample 500 times and finally loaded it ten times in active and inactive states (the test results presented in the figure are for the final ten cycles). The initial and final inactive tests showed very good compatibility except for a shift in the initial elongation of the sample at zero force (from 0 to $20 \%$ strain). The shift in the no-load strain is mainly caused by unrecoverable strain in the SMP layer and by its relative movement in silicone. Most of the unrecoverable strain happens in the first few cycles and the shift in the stress-strain diagram from tenth cycle on is rather negligible.

The test results confirms repeatable deformation of ASL in more than 500 cycles and almost 4 times drop in its stiffness in active state. The ratio of stiffness in active and inactive states can be controlled by using silicone rubbers with 


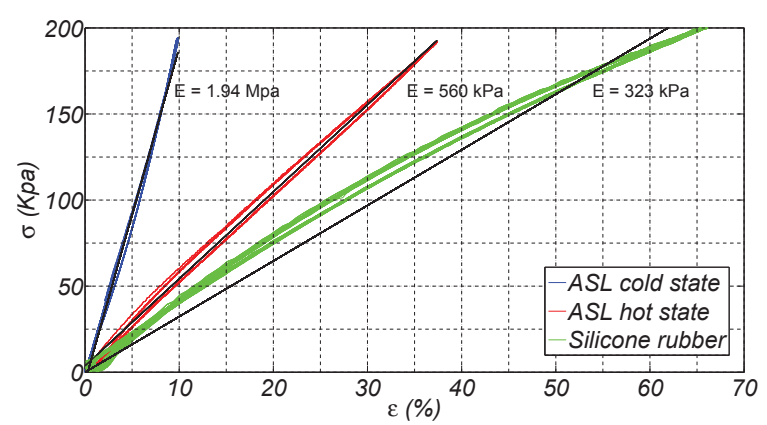

Fig. 4: Result of the characterization tests on a silicone rubber sample and an ASL in active and inactive states. The ASL is composed of SMP and heaters (540 $\mu \mathrm{m}$ thick) layer embedded in $4 \mathrm{~mm}$ thick layer of Dragon skin 30 form Smooth-on. The temperature increase softens the ASL almost 4 times. Here, the stress strain behavior of silicone and ASL are approximated by a linear function.

different modulus of elasticity and also the relative thickness of the SMP and silicone layers. Here, we have chosen a very hard polymer and designed the ASL to have a rather thick silicone layer to maximize the strain recovery of the SMP layer. An important feature of the characterization test results is its linearity (especially for the complete ASL sample). This simplifies the design process which is presented in the next section. We also briefly studied the dependency of the ASL stress strain behavior on the loading rate. This behavior was found not to be dependent on the speed in the three loading rates we have tested : $0.75 \% / s, 1.5 \% / s$, and $5 \% / s$ (the maximum possible loading speed). We should point out that the viscoelasticity of the polymers is expected to effect the behavior of the actuator since its loading rate will be faster than what is achievable by the test setup.

\section{DESIGN AND FABRICATION OF SPA WITH ASLS}

SPAs are air chambers in compliant material. Upon application of air pressure, the chamber expands. Based on the desired motion, layers with higher stiffness are embedded in selected areas of the chamber. This channels the compressed air to generate deformation and force in the desired direction. In this research, we replace the stiffer layer with an adjustable stiffness layer. The goal as presented in Fig. 1 is to generate multi modes of actuation in an SPA with a single chamber. The design of the SPA with ASL and its fabrication process are presented in the following sections.

\section{A. Design and performance estimation}

Actuator design parameters are determined using the characterization test results (from Section II-C) and the requirement to achieve $90^{\circ}$ angle in actuation in mode $\mathrm{A}$ and B (Fig. 1). We consider as the maximum allowed strain in the ASL, the maximum strain applied in the characterization test $(36 \%)$, which was highly repeatable. Fig. 5 presents a schematic of the actuator as it bends. Considering a uniform radius of curvature throughout the length, we will have the following relation for the strain in the ASLs $\left(\varepsilon_{h}\right.$ and $\left.\varepsilon_{c}\right)$, the bending angle $(\theta)$, and the length and height of the chamber ( $l_{\text {chamber }}$ and $\left.h_{\text {chamber }}\right)$ :

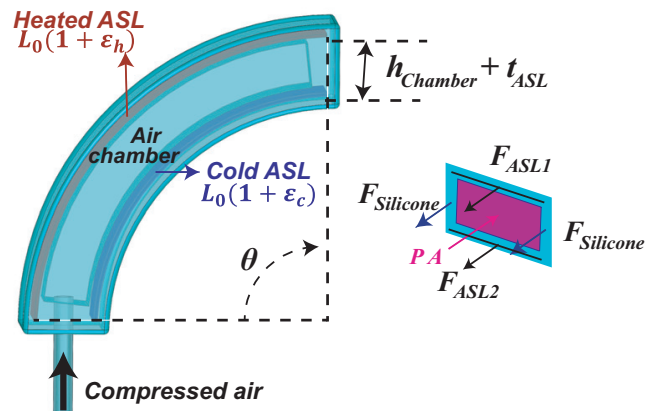

Fig. 5: Schematic of a SPA actuator with embedded ASLs. The figure shows the actuator in the deformed state and its design parameters. Also, forces applied to the cross section of the actuator are presented. In free deformation of the actuator, the force generated by the compressed air are transformed to stress and hence strain in the chamber walls. Different stiffness (controlled by the temperature) determines the strain in different walls of the chamber and hence the overall deformation.

$$
\left(\varepsilon_{h}-\varepsilon_{c}\right) l_{\text {chamber }}=h_{\text {chamber }} \theta_{\text {goal }}
$$

Based on (3), smaller the chamber height, shorter its length needs to be for reaching a goal angle while staying in the ASL allowed range of strain. On the other hand, a chamber with smaller height generates smaller blocked force (since the cross section area is smaller). Since we do not have any requirement on the blocked torque of the actuator in this research, the height of the chamber remains an open parameter. Based on the previous experience, we often put the sum of the wall thickness on the two sides of the chamber as the lower limit on the chamber height. We confirmed through experiments that to have a leak-free chamber, the thickness of the walls should be bigger than $4 \mathrm{~mm}$. This is bigger than the minimum wall size for normal SPAs since

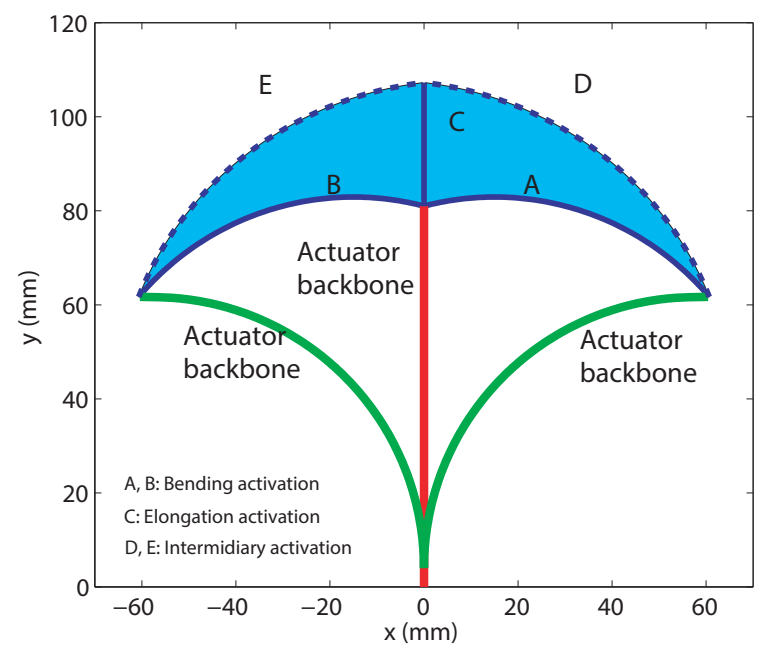

Fig. 6: The workspace of the actuator. The red line represents the initial state of the actuator, the green lines represent actuator as it bends to the side. The shaded area represents the overall work space of the actuator. the solid blue lines represent the 3 major activation modes (bending to the opposite sides and elongation). The dashed lines present the intermediary activation modes. 


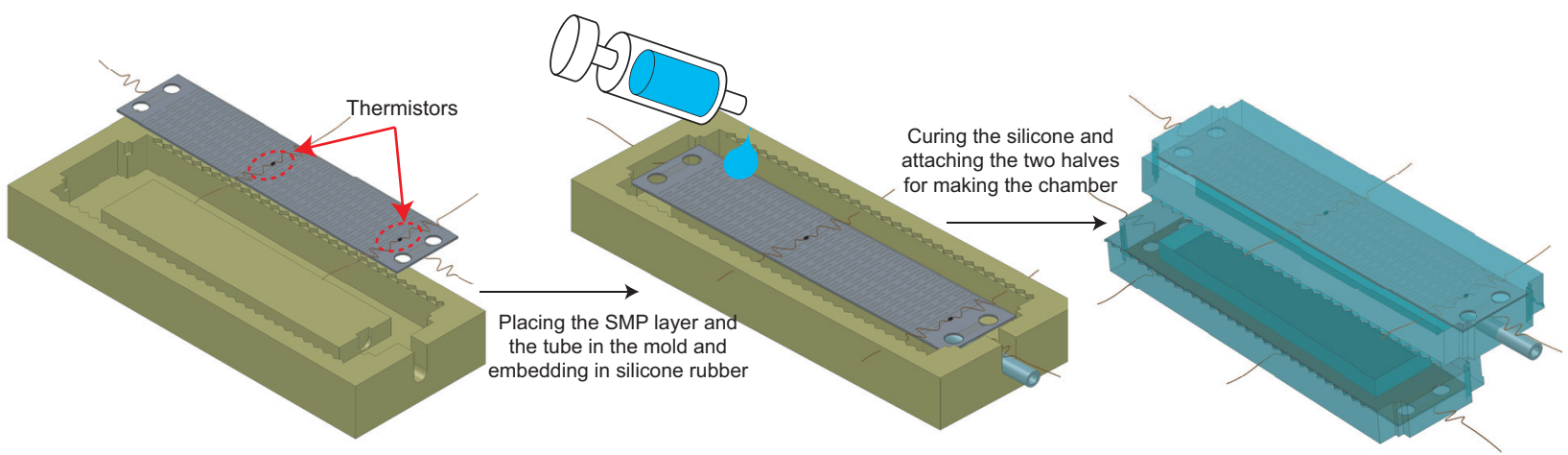

Fig. 7: The fabrication process of the SPA with embedded ASL. Embedding of the SMP layers in silicone is similar to the process explained for the test sample. After curing the silicone, the two halves of the actuator are glued together with silicone adhesive and thread is wrapped around the actuator to confine the lateral inflation of the actuator.

the walls in this design are composites materials with risk of delamination. Based on these, we have designed the chamber height to be $9.5 \mathrm{~mm}$ which places the SMP layers $12.5 \mathrm{~mm}$ apart, given the thickness and placement of the SMP layers in ASL. For a chamber of this size and given the maximum allowed strain in the ASL, the minimum chamber length for reaching to the desired range of motion is calculated according to (3) to be $73 \mathrm{~mm}$.

The width of the chamber is also dependent on the required blocked force and in this research remains an open parameter. The only limitation on the chamber width is that it should be smaller than the width of the SMP layer. We used the same width for the SMP layer as used in the characterization tests $(20 \mathrm{~mm})$. Through experiments, we confirmed that making the chamber $2 \mathrm{~mm}$ slimmer on each side prevents leaks (that makes the overall width of the chamber $16 \mathrm{~mm}$ ).

Fig. 5 presents the forces in a cross section of the actuator. Here, we estimate the pressure needed for generating the desired deformation, given the chamber size and ASL stiffness. In an actuator with free displacement, the force generated by the compressed air is entirely spent on deformation of the chamber walls.

$$
P A_{C h}=F_{A S L 1}+F_{A S L 2}+F_{\text {Silicone }}
$$

Based on (4) and the characterization results presented in Fig. 4, the pressure required for reaching the maximum deformation angle is estimated to be $330 \mathrm{kPa}$. We should point out that the contribution of the thread that wraps around the actuator and the lateral expansion of the actuator has been neglected in this study.

The workspace of a normal SPA is limited to a $1 \mathrm{D}$ trajectory. In the case of the actuator introduced here, the work space covers three major lines corresponding to: activation of each individual ASL (bending in mode A and B) and activation of the both ASLs (elongation in mode C). Moreover, by incremental control of the temperature (hence the stiffness difference between the two ASLs), deformation in the intermediary modes between the three major activation modes is possible (a combination of bending and elongation in the actuator). Fig. 6 presents the estimated work space of the actuator based on the following relation between the length of each ASL $\left(L_{A S L 1}\right)$, the bending angle $(\theta)$, and the curvature radius $(\rho)$ of the actuator:

$$
\begin{array}{r}
\Theta=\frac{L_{A S L 1}-L_{A S L 2}}{h_{\text {chamber }}} \\
\Theta=\frac{\left(L_{A S L 1}+L_{A S L 2}\right) / 2}{\Theta}
\end{array}
$$

Considering the forces depicted in Fig. 5, at the equilibrium point, the forces in the two ASLs should be equal (considering these the main contributors) and using characterization test results in Fig. 4 we can estimate the relative length of the two ASLs and from it, the actuator tip point position and orientation. In Fig. 6, the three solid blue lines present the main modes of actuation: bending toward sides and elongation. When compressed air flows into the chamber of an actuator with one ASL in soft and the other one in completely stiff state, the actuator bends along line A or B (depending on which ASL is activated) according to the input pressure. By activating the cold ASL partially, the actuator elongates and bends at the same time. The dashed lines (D and E) in Fig. 6 present the possible tip points when SPA is actuated at maximum pressure and the inactive ASL is heated incrementally to completely active state. In this figure, line $\mathrm{C}$ corresponds to the work space of the SPA with both ASLs in active state which causes elongation in the actuator. Theoretically, with a controlled and uniform temperature throughout the SMP layer, all the points in the shaded area of Fig. 6 can be accessed.

\section{B. Fabrication of SPA with embedded ASL}

The fabrication of the SPA with ASL starts with embedding the heater in SMP and cutting its outline which is the same as the fabrication of the test samples. Two thermistors are attached to each SMP layer to provide temperature feedback. The SPA is composed of two half sections that make the chamber as presented in Fig. 7. The holders in the mold keep the SMP layer in the proper distance from the chamber wall to avoid leaking. After the two halves are cured, they are attached together using silicone adhesive. 


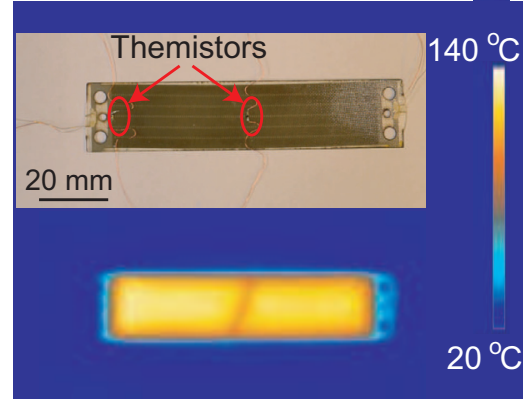

Fig. 8: Thermistors' position on the SMP layer and temperature distribution on the SMP surface. The thermal image shows considerable temperature gradient in the SMP layer. To ensure that the whole material has entered the rubbery state, we need to put the set temperature higher than the glass transition temperature (in this study, 110 instead of $55^{\circ} \mathrm{C}$ ).

Finally, we wrap a thread around the actuator using the teeth marks, left by the features designed in the mold, as the guide.

\section{RESULTS AND DISCUSSION}

In this section, the result of the performance tests on the SPA with ASL are presented in two parts. First, we study the effectiveness of the temperature control and temperature distribution in the SMP layer and then, the results of actuating SPA in different modes are presented.

\section{A. Temperature gradient and transient temperature in SMP}

One of the states of the contribution of the present work in respect to previous research [13], is the use of thermistors in contact with the thermoplastic SMP layer (as presented in Fig. 8). One of the critical aspects in thermally activated polymeric materials is heat distribution. Nonuniform temperature distribution results in mixed behaviors such as plastic deformation and overstrain in segments of the SMP layer causing unrecoverable strain. As presented in Fig. 8, there is a considerable temperature gradient for the sample with the present heater design. To study the temperature distribution, two thermistors in contact with each SMP layer were embedded in the actuator. The use of the sensors at the border, is useful for ensuring that the rubbery state is

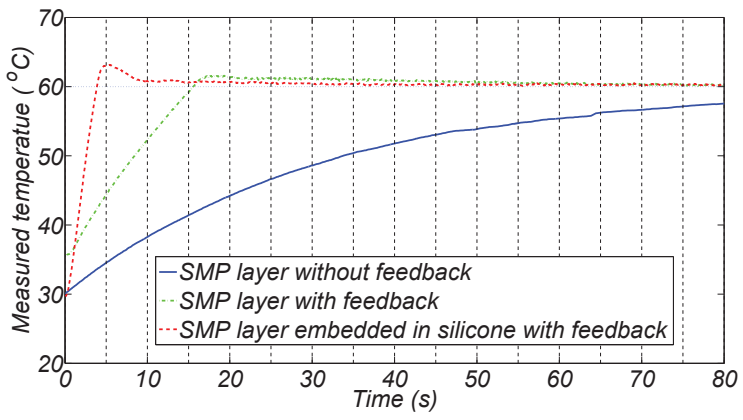

Fig. 9: Temperature of the SMP layer for 3 experiments: SMP layer with and without feedback, and SMP layer embedded in silicone with temperature feedback. Using a PI controller with the temperature feedback reduces the rise time from 120 second to around 5 seconds for the SMP sample outside the silicone. Embedding the sample in silicone increases the rise time since more energy is required to heat up the silicone in contact with SMP.

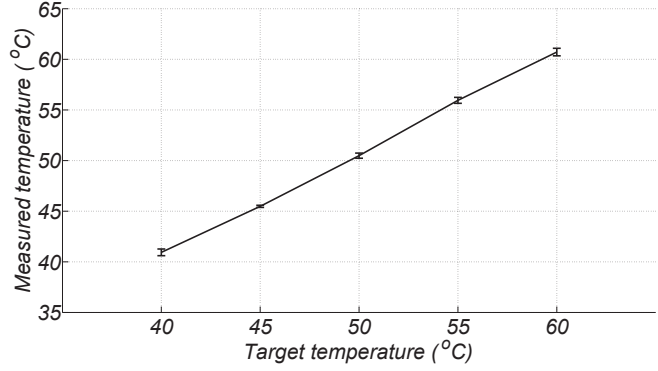

Fig. 10: SMP temperature (from the thermistor) versus the set temperature The graph shows good accuracy and precision (presented as the error bars in the graph) in following the temperature which means incremental activation between the major modes of operation in SPA would be possible.

reached all over the SMP layer. Moreover, studying the temperature distribution using multiple embedded sensors helps with future designs for the heater for having a more uniform distribution of temperature. A temperature drop from 10 to $20{ }^{\circ} \mathrm{C}$ has been evaluated and has been taken into account for defining the target temperature of the central part $\left(100-120{ }^{\circ} \mathrm{C}\right)$ for the tests on the SPA in order to ensure complete transition into rubbery state.

The feedback from the thermistors allows us to close the loop for maintaining a desired temperature. Adding feedback has the advantage of compensating any change in the ambient temperature or heat exchange rate. Moreover, a simple proportional-integrative (PI) controller, reduces the rise time considerably. Fig. 9 presents a comparison between the transient response of the temperature of a SMP layer for two cases: first when the fixed voltage needed to maintain a certain steady state temperature is applied to the heater, and second when a PI controller with feedback from thermistors controls heater's driving voltage. The results clearly shows the advantage of using the feedback control by reducing the rise time from around $120 \mathrm{~s}$ to around $5 \mathrm{~s}$. This figure also presents the transient temperature of a SMP layer embedded in silicone. Embedding the SMP layer in silicone increases the rise time considerably (from 5 to $20 \mathrm{~s}$ ), given the higher energy required for heating up the silicone in contact with the SMP layer. We confirmed through experiments (Fig. 10) that embedding the SMP layer in silicone does not affect the accuracy of the temperature control and precise (with standard deviation of less than $0.5^{\circ} \mathrm{C}$ ) and accurate (with less than $1{ }^{\circ} \mathrm{C}$ steady error) operating temperature was achieved which guarantees a fine control of the SMP temperature and its stiffness. This is important since it enables us to activate SPA in intermediary modes.

\section{B. Actuating different DoFs in SPA with ASL}

To study the performance of the SAP with embedded ASLs, we tested the actuator under different thermal conditions with different input pressures. The goal is to determine the work space of the actuator and compare it with the estimated work space. Table I presents the test condition used in actuator characterization. These tests are designed to determine the borders of the work space and the test 


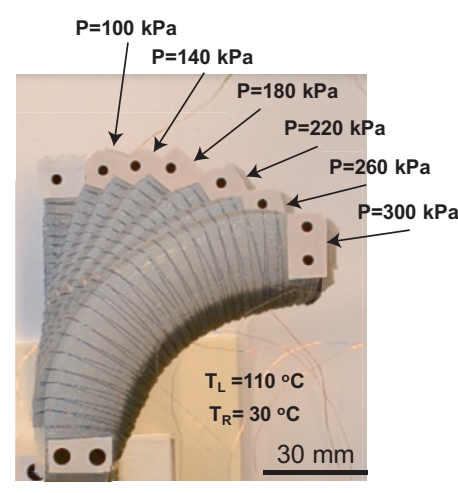

(a)

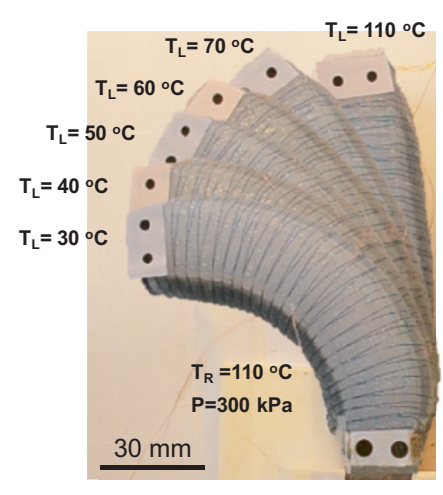

(b)

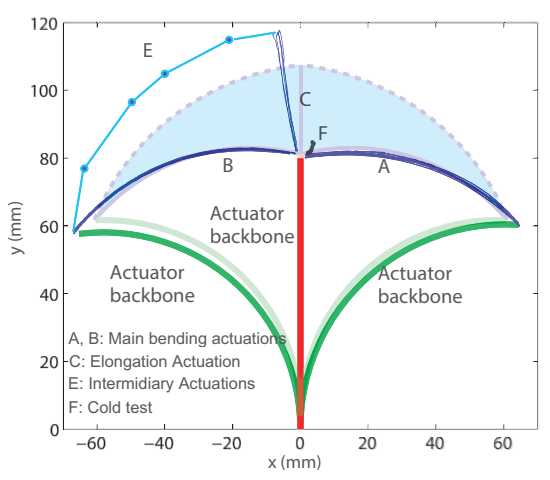

(c)

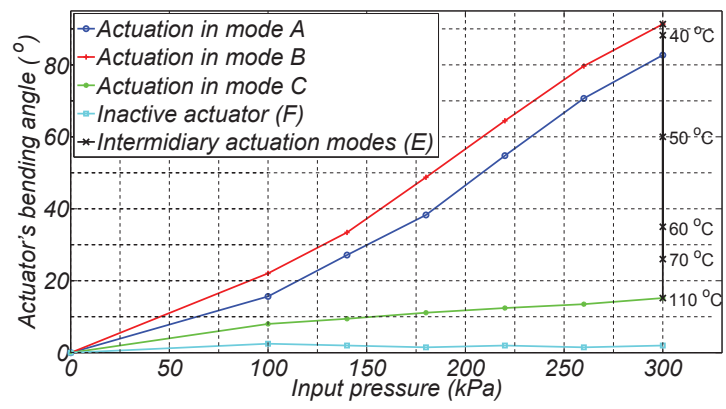

(d)

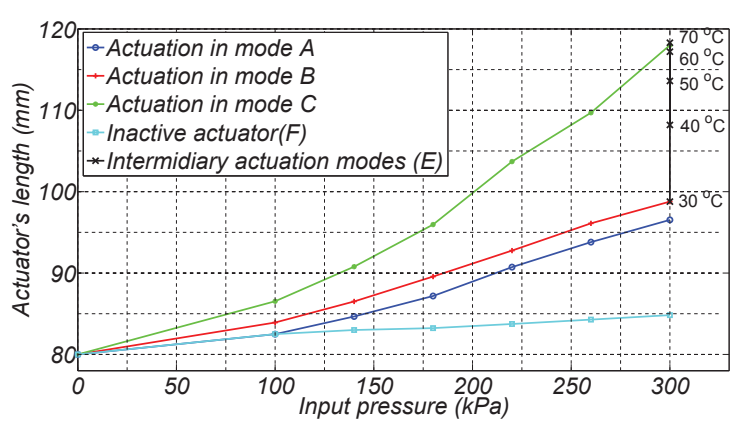

(e)

Fig. 11: The test results for the SPA with embedded ASLs. Actuation of SPA with one ASL activated (at $110{ }^{\circ} \mathrm{C}$ ) and the second one inactive causes bending in the actuator with bending angle being a function of the input pressure (a). Actuating the SPA at maximum pressure with one ASL active, causes bending but as we gradually increase the temperature of the second ASL, the actuator starts to elongate (b). The workspace of the actuator covers an area similar to the expected work space (a faded view of the estimated work space is overlapped for comparison). In high pressures, due to the lateral expansion of the actuator which increases the force generated by the compressed air, the length is longer than expected which causes the discrepancy between the experimental and estimated work spaces. This effect is more noticeable when both ASLs are active (lines $\mathrm{C}$ and $\mathrm{E}$ ) (c). The bending angle of the actuator versus pressure for different modes of activation (d). The small difference between the bending angle for the two sides is caused by small differences in geometry of the two halves of the actuator caused by fabrication errors such as differences in the thickness of the SMP layers. The length of the actuator in different modes of activation shows increasing rate of change with pressure as the pressure increases (e). The reason is the lateral expansion of the chamber which amplifies the effect of the pressure increase.

reference in the table refers to the work space borders represent in Fig. 6. The maximum pressure in these tests was determined by increasing the input pressure for the actuator in bending (mode A and B) till reaching $90^{\circ}$ bending angle (the goal in the design section). The required pressure for this deformation was $300 \mathrm{kPa}$ which is lower than the expected pressure from Section III-A. The pressure in the design section was over estimated since the lateral expansion of the chamber was neglected. The lateral expansion increases the

TABLE I: Test conditions to characterize the main modes of operation and the borders of the work space. The pressure increased in $40 \mathrm{kPa}$ increments from 100 to $300 \mathrm{kPa}$ to characterize the main modes of operation (A, B, and $\mathrm{C}$ ). For intermediary modes, the pressure was set to maximum and the temperature was increased in $10^{\circ} \mathrm{C}$ steps from 40 to $70{ }^{\circ} \mathrm{C}(\mathrm{E})$. The last test is applying pressure to the inactive actuator.

\begin{tabular}{|c||c||c||c|}
\hline Test ref. & $T_{L}\left({ }^{\circ} \mathrm{C}\right)$ & $T_{R}\left({ }^{\circ} \mathrm{C}\right)$ & $P(\mathrm{kPa})$ \\
\hline $\mathrm{A}$ & 110 & 30 & $100-300$ \\
\hline $\mathrm{B}$ & 30 & 110 & $100-300$ \\
\hline $\mathrm{C}$ & 110 & 110 & $100-300$ \\
\hline $\mathrm{E}$ & $40-70$ & 110 & 300 \\
\hline $\mathrm{F}$ & 30 & 30 & $100-300$ \\
\hline
\end{tabular}

area of the cross section of the chamber and as a result, the force needed to generate large enough strain for reaching 90 $\circ$ is achieved in a lower pressure.

Fig. 11a presents the overlapped snapshots taken from actuation in mode $\mathrm{A}$. in this test, the input pressure changes from 100 to $300 \mathrm{kPa}$ in $40 \mathrm{kPa}$ increments while the temperature is fixed at 110 and $30^{\circ} \mathrm{C}$ for the ASL on the left and right respectively. Fig. $11 \mathrm{~b}$ presents the snapshots from intermediary modes of operation (E) in which the pressure is fixed at $300 \mathrm{kPa}$, the temperature of the right ASL is fixed at $110^{\circ} \mathrm{C}$, and the temperature of the left ASL changes from 30 to $70{ }^{\circ} \mathrm{C}$ in $10{ }^{\circ} \mathrm{C}$ increments. Using a tracker software, we determined the overall work space of the actuator from these tests (Fig. 11c). The shape of the work space is as expected (Fig. 6), but compared to the estimated work space, the length of the actuator is longer. This is more significant in higher pressures and specifically in tests where both ASLs are active (lines C and E). In the elongation test (C), the actuator length increases $52 \%$ of the chamber length. This is substantially higher than the expected $36 \%$. This extra increase in length can be attributed 
to the lateral expansion of the actuator which increases the force from the compressed air and hence the strain and elongation in the chamber walls. This is also the reason of the increasing slope of the angle and length graphs with increase in the pressure (Fig. 11d,11e). Based on the rather linear stress-strain behavior of the materials (Fig. 4), we expected a linear relation for bending angle and length of the actuator versus the input pressure. But as the pressure increases, so does the area of the chamber which causes nonlinear relation between the force exerted to the chamber walls and the input pressure. The thread that wraps around the actuator confines the lateral expansion considerably but the chamber still expands. Ideally, we expected the actuation in mode $\mathrm{A}$ and $\mathrm{B}$ to give identical result. But because of the discrepancies in the manufacturing (such as the variation in the thickness of the SMP layers), the results of actuation in these two modes are asymmetric. This is also the reason for small bending angle in actuation in mode C. Such issues can be accounted for in future by adjusting the activation temperature of the two ASLs.

\section{CONCLUSIONS AND FUTURE WORK}

Increasing the number of DoFs in robots driven by SPAs is dependent on miniaturization of SPAs or emending additional DoF in a single actuator. In the conventional SPAs, a chamber with independent pressure line is needed for each DoF. Given the limitations on the size of tubing and pressure distribution grid (necessary for providing enough air flow), reducing the size of the actuator or adding additional chambers with independent pressure input is a a rather challenging task beyond the current designs for SPAs. The additional equipment necessary for each independent pressure line can also be troublesome in applications where the overall size and weight are confined.

In this research, we proposed a method for actuating a SPAs with a single chamber in different modes by manipulating the stiffness of the chamber walls. We used modulus of elasticity change in SMP around its glass transition temperature to adjust the stiffness of the walls. We confirmed, through characterization tests, that the repeatable deformation in a composite of silicone rubber, SMP, and heater (introduced in this work as the adjustable stiffness layer (ASL)) without loss of functionality in more than 500 cycles is possible. The stiffness of the ASL decreases four times upon activation. We designed a SPA according to the characterization data to reach $90^{O}$ bending in opposite directions in two modes of activation and elongation in the third mode. In the design presented here, the temperature of ASLs can be controlled in increments using the feedback from embedded off-the-shelf thermistors. Based on this, the estimated work space also included the intermediary modes of actuation which considerably enlarges the workspace. The characterization tests on the final actuator confirmed the three modes of actuation as well as the intermediary modes of actuation. Due to the lateral expansion of the actuator, the test results showed some discrepancy with the estimated performance for the actuator but the overall behavior of the actuator was in accordance with the estimated one. Confining the lateral expansion with methods more effective than wrapping the thread around the actuator or including the lateral expansion in the model is a topic for the future work.

\section{ACKNOWLEDGMENT}

This work was supported by Swiss National Center for Competence in Research in robotics.

\section{REFERENCES}

[1] S. Yi, S. Yun Seong, and J. Paik, "Characterization of silicone rubber based soft pneumatic actuators," in Intelligent Robots and Systems (IROS), 2013 IEEE/RSJ International Conference on, pp. 4446-4453.

[2] S. Yun Seong, S. Yi, R. van den Brand, J. von Zitzewitz, S. Micera, G. Courtine, and J. Paik, "Soft robot for gait rehabilitation of spinalized rodents," in Intelligent Robots and Systems (IROS), 2013 IEEE/RSJ International Conference on, pp. 971-976.

[3] M. Wehner, B. Quinlivan, P. M. Aubin, E. Martinez-Villalpando, M. Baumann, L. Stirling, K. Holt, R. Wood, and C. Walsh, "A lightweight soft exosuit for gait assistance," in Robotics and Automation (ICRA), 2013 IEEE International Conference on, pp. 3362-3369.

[4] J. M. Florez, B. Shih, Y. Bai, and J. Paik, "Soft pneumatic actuators for legged locomotion," in IEEE International Conference on Robotics and Biomimetics.

[5] R. F. Shepherd, F. Ilievski, W. Choi, S. A. Morin, A. A. Stokes, A. D. Mazzeo, M. W. Xin Chen, and G. M. Whitesides, "Multigait soft robot," Proceedings of the National Academy of Sciences, 2011.

[6] K. C. Galloway, P. Polygerinos, C. J. Walsh, and R. J. Wood, "Mechanically programmable bend radius for fiber-reinforced soft actuators," in Advanced Robotics (ICAR), 2013 16th International Conference on. IEEE, pp. 1-6.

[7] R. V. Martinez, C. R. Fish, X. Chen, and G. M. Whitesides, "Elastomeric origami programmable paper-elastomer composites as pneumatic actuators," Advanced Functional Material, vol. 22, pp. 1376-1384, 2012.

[8] E. Steltz, A. Mozeika, N. Rodenberg, E. Brown, and H. M. Jaeger, "Jsel: Jamming skin enabled locomotion," in Intelligent Robots and Systems, 2009. IROS 2009. IEEE/RSJ International Conference on, pp. 5672-5677.

[9] N. G. Cheng, M. B. Lobovsky, S. J. Keating, A. M. Setapen, K. I. Gero, A. E. Hosoi, and K. D. Iagnemma, "Design and analysis of a robust, low-cost, highly articulated manipulator enabled by jamming of granular media," in Robotics and Automation (ICRA), 2012 IEEE International Conference on, pp. 4328-4333.

[10] M. Cianchetti, T. Ranzani, G. Gerboni, I. De Falco, C. Laschi, and A. Menciassi, "Stiff-flop surgical manipulator: Mechanical design and experimental characterization of the single module," in Intelligent Robots and Systems (IROS), 2013 IEEE/RSJ International Conference on, pp. 3576-3581.

[11] K. Takashima, J. Rossiter, and T. Mukai, "Mckibben artificial muscle using shape-memory polymer," Sensors and Actuators A: Physical, vol. 164, no. 12, pp. 116-124, 2010.

[12] K. Takashima, T. Noritsugu, J. Rossiter, S. Guo, and T. Mukai, "Development of curved type pneumatic artificial rubber muscle using shape-memory polymer," in SICE Annual Conference (SICE), 2011 Proceedings of, pp. 1691-1695.

[13] T. Kazuto, S. Kazuhiro, M. Naohiro, S. Seiya, N. Toshiro, and M. Toshiharu, "Pneumatic artificial rubber muscle using shapememory polymer sheet with embedded electrical heating wire," Smart Materials and Structures, vol. 23, no. 12, p. 125005, 2014.

[14] A. Firouzeh, S. S. M. Salehian, A. Billard, and J. Paik, "An under actuated robotic arm with adjustable stiffness shape memory polymer joints," in Robotics and Automation (ICRA), 2015 IEEE International Conference on (To Appear).

[15] T. Hisaaki, H. Hisashi, Y. Etsuko, and H. Shunichi, "Thermomechanical properties in a thin film of shape memory polymer of polyurethane series," Smart Materials and Structures, vol. 5, no. 4, p. 483, 1996.

[16] L. Hines, V. Arabagi, and M. Sitti, "Shape memory polymer-based flexure stiffness control in a miniature flapping-wing robot," Robotics, IEEE Transactions on, vol. 28, no. 4, pp. 987-990, 2012. 